\title{
AMBIVALENCE. LUCI E OMBRE DEL LAVORO DIGITALE
}

Jel Classification: E24, E26, 033

\author{
di Francesca Coin, Marco Marrone
}

\begin{abstract}
Nel 2013 Trebor Scholz curava per Routledge un testo titolato Digital Labor: The Internet as Playground and Factory. Il testo raccoglieva i contributi di alcuni tra gli studiosi più innovativi e eclettici del lavoro digitale e si proponeva di evidenziarne le tendenze e le implicazioni nell'epoca contemporanea. Il tema era il cambiamento del lavoro mediato dalle tecnologie di rete, nel momento in cui la tecnologia ibrida ogni ambito dell'esistenza sino a cambiare la definizione stessa di lavoro. Il dibattito evidenziava alcuni nodi irrisolti dell'economia digitale, in primo luogo i temi della rendita e del lavoro gratuito nell'ambito della rete, quali espressione della capacità di estrarre informazioni da milioni di consumatori come condizione per la difesa di posizioni strategiche nel capitalismo delle piattaforme. Il presente contributo si concentra precisamente su questi temi, sulla tendenza recente a interpretare la rete come un apparato di cattura distopico, a differenza delle speranze che le venivano associate, sottolineando le trasformazioni e il ruolo ambivalente svolto dall'automazione e della tecnologia nell'immaginario collettivo.
\end{abstract}

Parole chiave: economia digitale, lavoro digitale, lavoro non pagato, gig economy, quarta rivoluzione industriale, automazione, robotizzazione, capitalismo delle piattaforme

\section{AMBIVALENCE. IN THE SHADOWS OF DIGITAL LABOR}

In 2013 Trebor Scholz edited a book titled Digital Labor: The Internet as Playground and Factory (Routledge). The book collected contributions by some of the most innovative and eclectic scholars of digital work and highlighted the most important trends and implications of digital labor in the current times. The main theme was the transformation of labor mediated by the digital technologies, at a time when technology hybridises every aspect of existence. The debate highlighted some unresolved issues in the debate concerning the digital economy, first and foremost the role of rentiers and unpaid labor within the digital technologies, as an expression of the ability to extract information from millions of consumers and to use it as a condition for defending strategic positions in the market. This paper focuses precisely on the recent transformations and interpretations of the digital technologies, and analyses the tendency to interpret the net as a dystopian device, underlining the ambivalent role played by automation and technology in the social imaginary.

Key words: Digital Economy, Digital Labor, Free Labor, Gig Economy, Fourth Industrial Revolution, Automation, Robotization, Platform Capitalism 


\section{Introduzione}

Nel 2012 Trebor Scholz curava per Routledge un testo titolato Digital Labor: The Internet as Playground and Factory. Il testo raccoglieva i contributi di alcuni tra gli studiosi più innovativi e eclettici del lavoro digitale e si proponeva di evidenziarne le tendenze e le implicazioni nell'epoca contemporanea. Il tema era il cambiamento del lavoro mediato dalle tecnologie di rete, nel momento, cioè, in cui la tecnologia ibrida ogni ambito dell'esistenza sino a cambiare la definizione stessa di lavoro. Di fatto, il dibattito si inserisce all'interno di un filone di studi che ha aiutato a mettere a fuoco alcuni aspetti dell'economia digitale, in primo luogo il flusso di dati che ogni giorno fluiscono nella rete trasformando le interazioni tra utenti nella fonte di rendita di piattaforme digitali che somigliano sempre più a monopoli, come li ha definiti Jonathan Taplin (2017). L'ultima fase dell'analisi sociale del lavoro digitale, in questo senso, sembra essersi soffermata soprattutto sui temi della rendita e del lavoro gratuito nell'ambito della rete (Armano, Murgia, Teli, 2017), le modalità con cui la capacità di estrarre informazioni da milioni di consumatori non remunerati consente ai monopoli della rete di raccogliere gratuitamente e vendere sul mercato strumenti di profilazione di utenti e consumatori capaci di forgiare il consumo (Armano, Murgia, Teli, 2017; Terranova, 2000). La recente tendenza distopica nell'interpretazione della rete come un apparato di cattura invasivo e pervasivo dal punto di vista del controllo e della privacy, non toglie che la relazione tra la società e la tecnologia digitale abbia una storia assai lunga, che da decenni attribuisce all'automazione e alla tecnologia un ruolo centrale ancorché ambivalente nell'immaginario collettivo.

Di fatto, il modo in cui il digitale, l'automazione e la robotica trasformano l'organizzazione del lavoro è sempre stato interpretato in modo ambivalente. Negli anni Novanta, il tema della tecnologia faceva da perno all'immaginario di un futuro fondato sulla "Fine del lavoro", per citare il noto testo di Rifkin (1995), inaugurando un filone sociologico destinato ad avere molta influenza negli anni successivi e che in ultima istanza richiamava, sebbene con evidenti differenze di prospettiva e metodo, un immaginario post-lavorista come quello articolato in testi classici quali il Frammento sulle Macchine di Marx, o, da una prospettiva teorica ancora distinta, Prospettive economiche per $i$ nostri nipoti, dal titolo della conferenza tenuta da John Mynard Keynes nel 1930 a Madrid. Da Postcapitalismo di Paul Mason (2015) a Nick Srnicek e Alex Williams Inventare il futuro. Per un mondo senza lavoro (2016, trad. it. 2018), autori diversi negli anni successivi hanno rievocato l'immaginario di un futuro "senza lavoro", nel quale la compatibilità virtuosa tra tecnologia e società si risolve 
nell'esternalizzazione di interi settori produttivi all'automazione e alla robotizzazione. Una prospettiva alla quale sfuggiva, in modo un po' ingenuo bisogna dire, tanto l'analisi dei diversi gruppi d'interesse preposti a plasmare l'evoluzione del lavoro nella società contemporanea, quanto la critica femminista, che alla prospettiva della "fine del lavoro" ha sempre risposto sottolineando l'ineluttabile permanenza del lavoro di riproduzione (Del Re, 2012). Fatto sta che negli anni Novanta, la compatibilità virtuosa tra tecnologia e società si estrinseca nel fiorire di una letteratura tesa a osservare l'influenza liberatrice e per certi versi libertaria della tecnologia, dell'automazione, della robotizzazione e del digitale nella società occidentale. Il saggio di Richard Barbrook e Andy Cameron, Californian Ideology, forse più di tutti spiega come il neoliberalismo tecnologico della Silicon Valley degli anni Novanta descrivesse una sorta di utopia digitale nella quale la controcultura hippie della San Francisco degli anni Sessanta confluiva nella cybercultura. Nell'utopia digitale, dicevano Barbrook e Cameron, tutti saranno hip and rich. La rete offrirà la modalità prima di realizzazione della democrazia trasformando le nuove tecnologie in una sorta di "agorà elettronica" capace di creare stampa alternativa e radio indipendenti, in quello che secondo gli autori rappresentava "il primo passo per implementare la democrazia diretta dentro tutte le istituzioni" (Barbrook, Cameron, 1996: 47). Il cyberspazio, in questa letteratura, veniva interpretato come uno spazio di democrazia diretta capace di disintermediare lo Stato, stimolando l'entusiasmo libertario della Californian Ideology, in una prospettiva di autogoverno e libertà.

Negli ultimi anni, la pervasività della rete scandita da processi di controllo favoriti dai Big Data e dal Data Mining ha influenzato una curvatura gradualmente distopica dell'interpretazione della relazione tra tecnologie e società. I momenti chiave nella scansione cronologica di questo processo possono essere ricondotti al 2008 quando, secondo un rapporto di McKinsey, il numero di devices connessi ha superato la popolazione mondiale. Nel 2013, poi, il numero di individui connessi attraverso il telefono cellulare ha superato le connessioni da desktop (McGrane, 2013). Da allora, la vita quotidiana è stata catturata all'interno della rete dando luogo a un processo di ibridazione che ha portato su una nuova scala le prospettive dell'economia on demand e dell'Industria 4.0.

Il recente testo curato da Emiliana Armano, Annalisa Murgia e Maurizio Teli dal titolo Platform capitalism e confini del lavoro negli spazi digitali (Mimesis/Eterotopie, 2017) mostra precisamente come:

«gli immaginari costruiti socialmente intorno alla rete siano passati da un orizzonte tecno-utopico di esplorazione, allargamento delle conoscenze e apertura, tipico degli anni Novanta - quello degli in- 
cantati dalla rete - all'immaginario attuale, di segno opposto, fatto di inclusione selettiva e precarizzazione diffusa, che caratterizza il secondo decennio degli anni 2000, attraversato da una profonda crisi economica, dal ripiegamento dei desideri e da sentimenti di paura» (Armano, Murgia, Teli, 2017: 8).

Il nodo del contendere, come evidenziato nel testo a cura di Trebor Scholz (2012), è la duplice relazione tra la dimensione monopolistica delle grandi corporazioni della rete e l'informalità del lavoro, che pare vieppiù articolato attraverso piattaforme di intermediazione di domanda e offerta in una specie di catena del valore globale nella quale l'individuo autonomo, oramai spogliato delle tutele del lavoro subordinato, flessibile, disponibile e "indipendente", diviene destinatario privilegiato dei rischi d'azienda e costretto a investire sempre più sulla propria performance per difendersi da una contrattualizzazione sempre più individualizzata e precaria.

L'informalità del lavoro è indubbiamente uno dei punti oscuri del lavoro digitale, sempre più esposto alla crescente capacità di sorveglianza delle nuove tecnologie e privato di una dimensione non produttiva anche nel tempo di non lavoro. $\mathrm{Nel}$ suo Labor in the global digital economy, Ursula Huws (2014) osserva non a caso come il punto nella digital economy sia precisamente il cambiamento del lavoro in sé, ivi intendendo il passaggio da una condizione di lavoro subordinato e, come previsto dalla legislazione del Novecento, tutelato, a un insieme di gig - piccole performance come si soleva dire nel lessico musicale del Jazz da cui proviene la dicitura gig economy (Friedman, 2014) - in un'estrazione di lavoro pagato a cottimo, fatto di mansioni quantificabili e standardizzate, nel quale la tutela del lavoratore lascia spazio alla valutazione della qualità e dell'output delle sue prestazioni. In questo contesto, il cambiamento qualitativo del concetto di lavoro rimanda a una riorganizzazione del processo produttivo che si serve delle tecnologie digitali per ridisegnare ogni aspetto del lavoro contemporaneo. A emergere sono nuovi sistemi di controllo e sorveglianza della prestazione, lo human resources analytics, che si concentra sull'identificazione degli indicatori maggiormente in grado di prevedere l'efficienza delle risorse umane, quantificandone continuamente la disponibilità o l'assenteismo, in modo tale da garantire un elevato return on investment. Al reclutamento $4.0 \mathrm{si}$ affianca la continua valutazione dell'output dei "fattori produttivi", in un processo che destruttura sempre più le tutele del lavoro per sostituirle con nuovi indicatori di performance e strumenti di monitoraggio in base ai quali il controllo della forza lavoro aumenta in maniera proporzionale all'arbitrio aziendale. 


\section{Valore, connessioni e intensificazione del lavoro nel capitalismo delle piattaforme}

L'esistenza di una dimensione sommersa dell'economia, composta da prestazioni occasionali che sfuggono alla regolazione formale, non costituisce una novità del nostro tempo, ma è da tempo sotto gli occhi di sociologi e economisti (Hart, 1973; Portes, Castells, 1989). Piuttosto, la novità introdotta dalle piattaforme, sempre più considerate come un nuovo modello di impresa, risiede nella capacità delle nuove tecnologie di organizzare tale porzione dell'economia (Huws, 2016). È in questa direzione che vengono utilizzate gran parte delle tecnologie digitali, in particolare gli algoritmi che vengono impiegati da un lato per maneggiare il petrolio del nostro tempo, ossia i Big Data (Srnicek, 2017), dall'altro per organizzare e disciplinare coloro che si rifugiano nell'informalità per fuggire la disoccupazione (Dyer-Whiteford, 2015). L'uso degli algoritmi risulta in grado di organizzare l'economia informale mettendo in relazione l'offerta di quei servizi che sino ad ora erano stati tenuti ai margini dal capitalismo industriale, a partire dal lavoro di cura (babysitter, badanti, ecc.), passando per i più classici imbianchini e tuttofare, fino a giungere ai riders delle piattaforme di food delivery, con una domanda. Dietro un processo di organizzazione dell'economia informale, si nasconde un campo di tensione determinato dalla pulsione costante verso la monopolizzazione di nuovi spazi di mercato e dal dispiegarsi di processi estrattivi (Huws, 2014). In questo contesto - messo in luce, più che dalla ricerca accademica che si è trovata spesso ad amplificare la retorica dello sharing, dal moltiplicarsi delle contestazioni per esempio nell'ambito del food delivering - emerge come il tratto distintivo dell'economia digitale dal punto di vista di chi vi opera, sia anzitutto la qualità dello sfruttamento - quei dispositivi che consentono di eludere la regolamentazione e di intensificare i processi estrattivi sino a produrre a un modello di impresa che appare sempre più problematico dal punto di vista del suo impatto sociale.

\section{La fuga dalle regole}

In questo contesto, a caratterizzare l'emergere di queste nuove piattaforme vi è la tendenza a sottrarsi dai vincoli dell'economia formale. Una pulsione, questa, che caratterizza il comportamento delle piattaforme sin dalla nascita. Secondo Srnicek (2017), esiste una continuità non solo tra le logiche dell'outsourcing e il modo in cui queste piattaforme eludono i vincoli fiscali e salariali, ma vi è anche una relazione diretta tra questi processi, che si fondano sulla capacità di creare economie di scala e di reinvestire 
il capitale accumulato dai processi di outsourcing in finanza e nello sviluppo delle nuove tecnologie. L'Economist (2016) ha, non a caso, descritto l'economia digitale come un processo caratterizzato dalla "nascita di superstars" - come si evince dal titolo di un articolo del 2016 titolato The rise of the superstars - evidenziando come la nuova economia digitale si differenzi dai processi di concentrazione di capitale tipici dell'epoca fordista precisamente per la capacità di ridurre il personale e di accrescere i profitti attraverso economie di scala. Il dato più rilevante, in questo senso, evidenzia come le principali compagnie digitali di Silicon Valley nel 2014 occupassero un totale di 137 mila persone a fronte dell'1,2 milioni di persone occupate nel 1990 dalle tre principali compagnie automobilistiche degli Stati Uniti (The Economist, 2016). I profitti dell'innovazione tecnologica in questo contesto non vengono reinvestiti al fine di creare lavoro o reddito ma usati al fine di difendere la propria posizione strategica a descrivere un mercato digitale caratterizzato da una forte competizione tra le piattaforme stesse (Huws, 2016). Il risultato è quello di un mercato particolarmente dinamico e denso di innovazione tecnologica, ma definito anche dalla crescita esponenziale del numero di acquisizioni e fusioni - basti pensare all'acquisizione di What'sApp, azienda che vanta 1,3 miliardi di utenti connessi ogni giorno, da parte di Facebook per la cifra di 19 miliardi di euro nel 2014 - volte ad allargare il raggio di espansione dei propri servizi. Di fatto, la spinta verso il monopolio costituisce uno degli angoli più sporgenti delle piattaforme. La narrativa di frontiera delle piattaforme è quella di una great platform wars (Srnicek, 2017) e del master algorithm (Domingos, 2015) nuovo sacro Graal della produzione, che pur se spesso rischia di concedere troppo all'analisi delle tendenze, ben restituisce la tensione che caratterizza i confini delle piattaforme (Armano, Murgia, Teli, 2017).

Così accade che le piattaforme riescano a produrre valore impiegando un numero di lavoratori che non ha precedenti nella storia, quanto alla sua limitatezza numerica, mentre crea economie di scala caratterizzate dalla capacità di eludere i vincoli della redistribuzione fiscale e della retribuzione salariale. In questo contesto, la nascita di superstars rimanda a una manciata di imprese globali il cui giro d'affari è decuplicato in pochi anni e la cui posizione strategica dipende dall'estrazione di lavoro gratuito e dalla creazione di nuove catene di fornitura a chiamata.

Boltanski e Chiapello (1999) definiscono il nuovo spirito del capitalismo come "connessionista". Per certi versi una profezia quella dei sociologi francesi nei confronti della tendenza delle piattaforme non solo a incrementare il numero di connessioni, ma anche ad intensificare le transazioni della rete. Così, mentre le piattaforme di food delivery si basano su 
un marketing volto a incrementare il maggior numero degli account, i social network sviluppano sempre nuove funzioni con l'obiettivo di incrementare il tempo speso nella creazione di contenuti e Amazon si distingue per la capacità di imporre trasversalmente i propri servizi nel mercato dall'e-commerce al mechanical turk. In questo senso, pur se l'algoritmo che governa l'interazione con le piattaforme svolge a tutti gli effetti il ruolo del boss (Scholz, 2017), queste vengono condotte fuori dal rapporto salariale e nascoste dietro le retoriche del playbour (Lund, 2014), in un processo che cattura all'interno di nuove forme di socialità mentre legittima $\mathrm{i}$ tratti dell'informalità e del free work (Terranova, 2000).

\section{I dispositivi di valutazione della performance e l'intensificazione del lavoro}

Nel criticare la visione deterministica dell'impatto delle tecnologie sul lavoro che proviene da riflessioni come quelle di Frey e Osborne (2017), Pfeiffer evidenzia come a essere rilevante sia l'impatto sulla dimensione qualitativa della prestazione lavorativa (Pfeiffer, Suphan, 2015). In effetti, ciò che è in corso è un processo di intensificazione del lavoro (Huws, 2010) che, grazie a una sempre maggiore capacità di rilevare e processare dati, sollecita sempre più le performance dei lavoratori. A caratterizzare le piattaforme vi è infatti una trasversale diffusione di dispositivi di valutazione, a partire dall'uso del like, fino a produrre sistemi di ranking, che incidono direttamente sulle tutele e la sicurezza del lavoro. Il passaggio dall'inquadramento al ranking non solo inserisce il lavoro all'interno di logiche di gamification, funzionali all'occultamento del lavoro (Lund, 2014) e al suo inserimento all'interno di un sistema dato di regole volto a stabilire una governamentalità dei soggetti (Scharpe, 2014), ma divide i lavoratori che svolgono la stessa mansione sino a rappresentare un metodo diverso di inquadramento nel rapporto di lavoro. L'analisi dei dati delle prestazioni non è l'unico elemento che incide su questo meccanismo. A comporre il ranking vi sono infatti la quantificazione della prestazione, ma anche i feedback dei consumatori e il grado di conformazione degli individui ai profili selezionati dall'impresa. Paradossalmente, dunque, mentre da un lato le piattaforme tendono a eludere i vincoli della subordinazione, deresponsabilizzandosi nei confronti della forza lavoro, dall'altro viene richiesta una particolare responsabilità che porta a interiorizzare una particolare postura, incorporando dunque anche la spinta verso l'intensificazione della prestazione. 


\section{La disciplina della disconnessione}

Parallelamente alla spinta verso l'intensificazione emerge anche una ristrutturazione dei meccanismi di disciplinamento. A caratterizzare le piattaforme vi è infatti anche una particolare angolatura dell'inclusione differenziale che se da un lato seleziona quei profili biografici più facilmente riconducibili alla gig economy, quali i giovani, le donne, i disoccupati di lunga durata, ecc. (McKinsey\&Company, 2016), dall'altro esercita sugli individui un processo di imprinting (Chicchi, Leonardi, Lucarelli 2016) che finisce con il fornire alle piattaforme una soggettività particolarmente adatta a soddisfare i suoi bisogni organizzativi. In questo scenario, non solo lo spazio di autonomia viene sempre più ristretto, ma si fa strada anche un particolare processo di disciplinamento attraverso la possibilità di ricorrere da parte delle piattaforme alla disconnessione.

Si tratta di una logica di controllo che viene attivata ogni qualvolta si presenta un comportamento imprevisto presente tanto in quelle piattaforme che governano servizi come nel caso del delivering, ma anche in quelle che regolano le nostre attività riproduttive. Così, gli utenti che violano le regole possono vedere restringersi le opportunità di interazione e di connessione, quando non la sospensione del proprio account in modo temporaneo o definitivo. La capacità di funzionare sincronicamente dei dispositivi di valutazione della performance e quelli di disciplinamento finisce così con il mettere in campo un meccanismo contraddittorio che se da un lato sollecita gli individui verso la ricerca di visibilità, dall'altro li scoraggia da ogni forma di esposizione che possa mettere a nudo la propria fragilità contrattuale.

Pertanto, se da un lato le piattaforme sono caratterizzate da una bassa soglia di ingresso, consentendo un facile accesso alla possibilità di mettere a disposizione la propria capacità produttiva, dall'altro questa massa di lavoratori viene organizzata all'interno di specifiche interazioni che non tollera alcun possibilità di fuga. In un contesto dove le prestazioni, tanto di tipo produttivo, quanto di tipo riproduttivo, divengono possibili solo all'interno delle piattaforme stesse, la disconnessione costituisce così non solo un sostituto del licenziamento, ma anche un rafforzamento della pressione del ricatto. Essere disconnessi vuol dire infatti perdere la possibilità di svolgere "lavoretti", incrementando così i margini dell'intensificazione, prevenendone allo stesso tempo la possibilità di insubordinazione da questi meccanismi.

Negli ultimi mesi, tutti questi processi sono diventati particolarmente visibili nel caso dei riders. In un'ottima inchiesta per il Financial Times seguita allo sciopero di UberEats a Londra, Sarah O' Connor (2016) ha 
parlato di Taylorism on steroids per descrivere la pervasività della dataveillance esercitata sui riders da parte delle nuove tecnologie digitali, descrivendo la gig economy come un'ottima "opportunità per lo sfruttamento rapace" di persone che siedono già al fondo della scala sociale, come ha dichiarato Guy Standing (citato in Sarah O' Connor, 2016; Coin, 2017). Il lavoro digitale, in questo senso, pare la quintessenza del mercato contemporaneo: un sistema in cui l'obsolescenza della relazione salariale si risolve in una frammentazione delle prestazioni lavorative ridotte a un assemblaggio di occupazioni occasionali caratterizzate da un elevato controllo sulla performance e dalla costante riduzione delle tutele. In questo contesto, le speranze e l'immaginario utopico legati alle nuove tecnologie paiono gradualmente ridimensionarsi e tingersi di dimensioni oscure anzitutto nelle narrazioni dei lavoratori che si trovano loro malgrado a viverne le contraddizioni. Il caso dei riders e della food delivery, in questo senso, ha rappresentato forse la cartina tornasole più esplicita delle contraddizioni della gig economy e della capacità potenziale dei nuovi lavoratori on demand di trasformarsi nella leva prima della denuncia facendosi protagonisti di nuove forme di sciopero digitale e di contro-narrazioni destinate a plasmare dal basso il futuro del lavoro digitale.

\section{Riferimenti bibliografici}

Armano E., Murgia A., Teli M., a cura di (2017). Platform Capitalism e confini del lavoro negli spazi digitali. Roma: Mimesis.

Barbrook R., Cameron A. (1996). The Californian Ideology. Science as Culture, 6(1): 44-72.

Boltanski L., Chiapello E. (1999). Le nouvel esprit du capitalism. Paris: Gallimard.

Breman J., van der Linden M. (2014). Informalizing the Economy: The Return of the Soaicl Question at a Global Level. Development and Change, O(00): 121, doi: 10.1111/dech.12115.

Chicchi F., Leonardi E., Lucarelli S. (2016). Logiche dello sfruttamento. Oltre la dissoluzione del rapporto salariale. Verona: Ombre Corte.

Coin F., a cura di (2017). Salari Rubati. Economia politica e conflitto ai tempi del lavoro gratuito. Verona: Ombre Corte.

Del Re A. (2012). Questioni di genere: alcune riflessioni sul rapporto produzione/riproduzione nella definizione del comune. AG. About Gender. International Journal of Gender Studies, 1(1): 151-170, doi: 10.15167/22795057/ag.2012.1.1.14.

Domingos P. (2015). L'Algoritmo definitivo. Torino: Bollati Boringhieri.

Dyer-Whiteford N. (2015). Cyber-Proletariat: Global Labour in the Digital Vortex. London: Pluto Press. 
Frey C.B., Osborne M.A. (2017). The future of employment: how susceptible are jobs to computerisation? Technological Forecasting and Social Change, 114: 254-280, doi: 10.1016/j.techfore.2016.08.019.

Friedman G. (2014). Workers without employers: shadow corporations and the rise of the gig economy. Review of Keynesian Economics, 2(2): 171-188, doi: $10.4337 /$ roke.2014.02.03.

Hart K. (1973). Informal Income Opportunities and Urban Employment in Ghana. Journal of Modern African Studies, 11: 61-89.

Huws U. (2010). Expression and expropriation: The dialectics of autonomy and control in creative labour. Ephemera, Theory \& Politics in Organization, 10(3/4): 504-521 -- <http://www.ephemerajournal.org/sites/default/files /10-3huws.pdf.>.

Huws U. (2014). Labor in the global digital economy: The cybertariat comes of age. New York: New York University Press.

Huws U. (2016, October 17). Understanding the 'Gig Economy': The Political Economy of Platform Capitalism. Global Labor Research Center Public Speaking. Toronto -- <http://glrc.apps01.yorku.ca/events/understanding-thegig-economy/>.

Keynes J. M. (1930). Economic Possibilities for our Grandchildren. In: Keynes J.M. (1963) Essays in Persuasion. New York: W.W. Norton \& Co.: 358373.

Lund A. (2014). Playing, Gaming, Working and Labouring: Framing the Concepts and Relations. tripleC: Communication, Capitalism \& Critique. Open Access Journal for a Global Sustainable Information, 12(2): 735-801.

Mason P. (2015). Postcapitalism. A guide to our future. London: Allen Lane.

McKinsey\&Company. (2016, October). Independent work: Choice, necessity, and the gig economy -- <https://www.mckinsey.com/global-themes/employme nt-and-growth/independent-work-choice-necessity-and-the-gig-economy>.

McGrane K. (2013). The Rise of the Mobile-Only User. Harvard Business Review May 28.

O' Connor S. (2016). When your boss is an algorithm. Financial Times, 8 September.

Pfeiffer S., Suphan A. (2015). The Labouring Capacity Index: Living Labouring Capacity and Experience as Resources on the Road to Industry 4.0. Working Paper 2015 \#2, University of Hohenheim, Chair for Sociology.

Portes A., Castells M. (1989). World Underneath: The Origins, Dynamics and Effects of the Informal Economy. In: Portes A., ed. The Informal Economy: Studies in advanced and less developed countries. Baltimore: John Hopkins University Press: 11-37.

Rifkin J. (1995). The End of Work: The Decline of the Global Labor Force and the Dawn of the Post-Market Era. New York: Putnam Publishing Group.

Scharpe N. (2014). Gamification and governamentality. In: Fuchs M., Fizek S., Ruffino P., Schrape N., eds. Rethinking gamification. Lunenburg: Meson Press: 21-46. 
Scholz T. (2012). Digital Labor: The Internet as Playground and Factory. New York: Routedge.

Scholz T. (2017). Think Outside the Boss. In: Armano E., Murgia A., Teli M., a cura di. Platform Capitalism e confini del lavoro negli spazi digitali. Milano: Mimesis: $37-56$.

Srnicek N., Williams W. (2016). Inventing the Future: Postcapitalism and a World Without Work. London: Verso. Trad. it. di F. Gironi (2018). Inventare il futuro. Per un mondo senza lavoro. Nero Press Edizioni.

Srnicek N. (2017). Platform Capitalism. Cambridge: Polity Press.

Taplin J. (2017). Move Fast and Break Things: How Google, Facebook and Amazon Have Cornered Culture and What It Means For All Of Us. London: Pan Macmillan.

Terranova T. (2000). Free Labor: Producing Culture for the Digital Economy. Social Text, $18(2$ [63]): 33-58, doi: 10.1215/01642472-18-2_63-33.

The Economist. Special Report (2016). The rise of superstars. The Economist, 17 September -- <http://www.economist.com/news/special-report/217070 48small-group-giant-companiessome-old-some-neware-once-again-domina ting-global>. 